\title{
Zero-bias anomaly and Kondo-assisted quasi-ballistic 2D transport
}

\author{
A. Ghosh, M. H. Wright, C. Siegert, M. Pepper, I. Farrer, C. J. B. Ford, D. A. Ritchie \\ Cavendish Laboratory, University of Cambridge, Madingley Road, Cambridge CB3 OHE, United Kingdom
}

(Dated: November 18, 2018)

\begin{abstract}
Nonequilibrium transport measurements in mesoscopic quasi-ballistic 2D electron systems show an enhancement in the differential conductance around the Fermi energy. At very low temperatures, such a zero-bias anomaly splits, leading to a suppression of linear transport at low energies. We also observed a scaling of the nonequilibrium characteristics at low energies which resembles electron scattering by two-state systems, addressed in the framework of two-channel Kondo model. Detailed sample-to-sample reproducibility indicates an intrinsic phenomenon in unconfined 2D systems in the low electron-density regime.
\end{abstract}

PACS numbers: 72.25.-b, 71.45.Gm, 71.70.Ej

Zero-bias anomaly (ZBA) in the non-equilibrium characteristics of ballistic systems provides additional insight into the coupling mechanism of a conduction electron with its surroundings. For example, a maximum in the differential conductance $\left(d I / d V_{\mathrm{SD}}\right)$ at the Fermi energy $\left(E_{\mathrm{F}}\right)$ in case of tunnelling via magnetic impurities 1], or through artificially confined quantum dots 2, 3], has been explained by a Kondo-like antiferromagnetic coupling of the electron to localized impurity spin. Similar enhancement observed in clean quantum point contacts in semiconductor heterostructures has led to much controversy regarding the spin-structure and effects of lateral confinement in such systems [4]. On the other hand, in quasi-ballistic metallic nanobridges the ZBA shows in a local cusp-like minimum in $d I / d V_{\mathrm{SD}}$ at $E_{\mathrm{F}}$, which has been interpreted in terms of nonmagnetic two-channel Kondo (2CK) framework arising from the interaction of the electrons with local two-state atomic defects [ [5]. The interest in this lies in the prediction that in particle-hole symmetric case, such a model flows to a $T=0$ fixed point, giving rise to quantum critical behavior and nonFermi liquid effects [6, 7].

Investigation of nonequilibrium ballistic transport in spatially extended 2D systems is primarily impeded by greater scattering from background disorder. The small level spacing requires relatively larger sample dimensions, resulting in significant momentum relaxation during the course of transport. Even though recent nonequilibrium studies in mesoscopic 2D electron systems (2DES) have shown an unexpected ZBA at low background disorder [8], the mechanism of transport in this regime remains unclear. Here we report the experimental observation of a new and unexpected feature in the nonequilibrium quasiballistic 2D transport. At zero magnetic field in unconfined samples of mesoscopic dimensions, we find a strong zero-bias enhancement in $d I / d V_{\mathrm{SD}}$, which splits at very low $T(\lesssim 150 \mathrm{mK})$ by a gate voltage $\left(V_{\mathrm{g}}\right)$-dependent magnitude. This modifies the linear transport properties by introducing intermittent low-energy nonmonotonicity in the linear conductance $G\left(=d I / d V_{\mathrm{SD}}\right.$ at $V_{\mathrm{SD}}=0$, where $V_{\mathrm{SD}}$ is the source-drain bias), as a function of both $T$ and in-plane magnetic field $\left(B_{\|}\right)$. At low $V_{\mathrm{SD}}$, an intriguing scaling behavior of $d I / d V_{\mathrm{SD}}$ in $T$ is also observed, which indicates a 2CK-type scattering of electrons in these systems at low $T$.

The devices were fabricated from $\mathrm{Si} \delta$-doped GaAs/AlGaAs heterostructures. In order to minimize the disorder arising from Coulomb potential of ionized dopants, we used a thick layer $(\approx 80 \mathrm{~nm})$ of undoped AlGaAs spacer, and adopted a slow cooling procedure [8] to maximize the correlations in the donor layer. The asgrown mobility of the wafers range over $\mu \sim 1-3 \times 10^{6}$ $\mathrm{cm}^{2} / \mathrm{V} \mathrm{s}$, depending on the donor $\left(n_{\delta}\right)$ and electron $\left(n_{\mathrm{s}}\right)$ density. Over the experimental temperature range, the momentum relaxation rate was typically $\tau^{-1} \lesssim(0.1-1) \times$ $k_{\mathrm{B}} T / \hbar$, indicating a quasi-ballistic nature of the transport [9]. The elastic scattering length $\lambda \sim v_{\mathrm{F}} \tau \sim 10 \mu \mathrm{m}$, where $v_{\mathrm{F}}$ is the Fermi velocity, provided an upper cutoff to the device dimensions, restricting the number of elastic scattering events to very few or none. Measurement of $d I / d V_{\mathrm{SD}}$ was carried out using standard mixed low-frequency ac/dc technique with ac excitation $\ll k_{\mathrm{B}} T$ at each $T$ inside a top-loading dilution refrigerator with base electron temperature $\approx 32 \mathrm{mK}$.

Fig. 1(a), (b) and (c) show the $B_{\|}=0$ nonequilibrium characteristics of three samples with different dimensions and doping profile. We varied both the total geometrical area, as well as the aspect ratio in samples T $45\left(2 \times 8 \mu \mathrm{m}^{2}\right.$, $\left.n_{\delta} \approx 2.5 \times 10^{12} \mathrm{~cm}^{-2}\right)$, T46 $\left(5 \times 8 \mu \mathrm{m}^{2}, n_{\delta} \approx 1.9 \times 10^{12}\right.$ $\left.\mathrm{cm}^{-2}\right)$ and T48 $\left(5 \times 5 \mu \mathrm{m}^{2}, n_{\delta} \approx 0.9 \times 10^{12} \mathrm{~cm}^{-2}\right)$. The dimensions are chosen in order to ensure small singleparticle level spacing with $\Delta \epsilon_{\mathrm{x}}, \Delta \epsilon_{\mathrm{y}} \sim h^{2} / 8 m^{*} L^{2} \ll k_{\mathrm{B}} T$ for each $T$. While no consistent feature in $G$ as a function of $V_{\mathrm{g}}$ could be identified (not shown), the ZBA around $V_{\mathrm{SD}}=0$ is evident for all samples 10. We focus on some of the common features of the ZBA shown in Fig. 1: (1) At $\left|V_{\mathrm{SD}}\right| \lesssim 0.15-0.2 \mathrm{meV}, d I / d V_{\mathrm{SD}}$ shows an enhancement at all $V_{\mathrm{g}}$. This energy scale varies only weakly with $V_{\mathrm{g}}$, and also $\ll E_{\mathrm{F}}$ at all $n_{\mathrm{s}}$. (2) Over certain ranges of $V_{\mathrm{g}}$, the ZBA splits by $2 \Delta$, leading to a double-peak structure around $E_{\mathrm{F}}$. (3) $\Delta$ is oscillatory in $V_{\mathrm{g}}$, and when normalized for series conductance, in particular for $G \gtrsim 2 e^{2} / h$, 

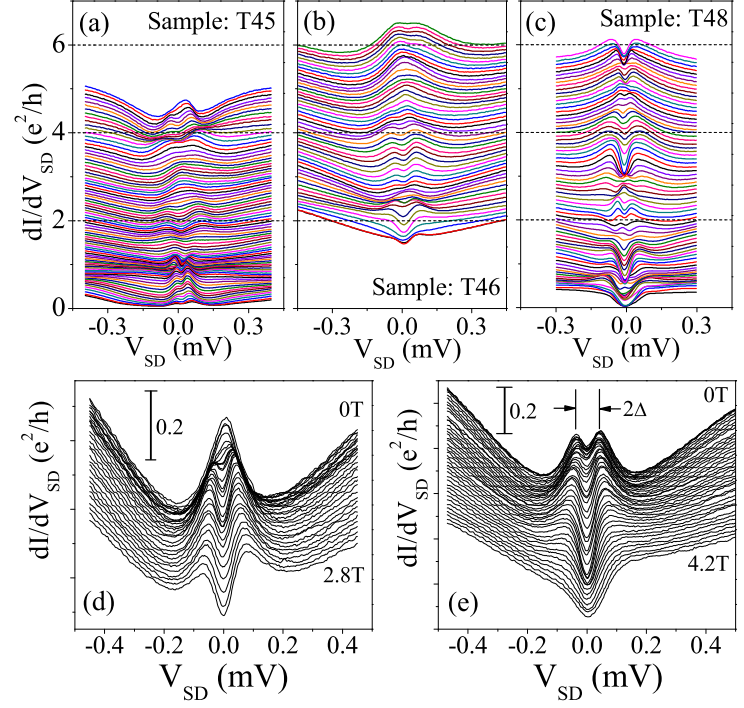

FIG. 1: (Color online) The zero-bias anomaly at $T \approx 32 \mathrm{mK}$ and $B_{\|}=0$ : (a)-(c) Nonequilibrium characteristics in three different samples. Each differential conductance trace is obtained for a fixed $V_{\mathrm{g}}$. (See text for sample details) (d),(e) $B_{\|}$-dependence of the nonequilibrium characteristic at the single peak and double peak regime $(T=32 \mathrm{mK})$. Traces are vertically shifted for clarity. The vertical bars denote a conductance change of $0.2 \times e^{2} / h$.

the splitting is strongest when $G$ lies close to an even integral multiple of $e^{2} / h$, becoming unresolvable around odd multiples. (4) While no clear dependence of the energy scales on size/shape of the samples was observed, the amplitude of the ZBA was found to depend on $n_{\mathrm{s}}$. Note that the ZBA is strongest in T48 $\left(n_{\mathrm{s}}=6.5 \times 10^{10}\right.$ $\mathrm{cm}^{-2}$ at $\left.V_{\mathrm{g}}=0\right)$, and weakest in T45 $\left(n_{\mathrm{s}}=10.1 \times 10^{10}\right.$ $\mathrm{cm}^{-2}$ at $\left.V_{\mathrm{g}}=0\right)$. Such weakening is also observed in a given sample as $V_{\mathrm{g}}\left(\right.$ or $\left.n_{\mathrm{s}}\right)$ is increased.

The single- and double-peak regions show distinct behavior in the presence of external in-plane magnetic field $\left(B_{\|}\right)$. As shown in Fig. 1(d) and (e), while the singlepeak splits by the Zeeman energy $\Delta_{\mathrm{Z}}=2 g^{*} \mu_{\mathrm{B}} B_{\|}$from $B_{\|}=0$, Zeeman splitting of the double peak appears only at relatively large (sample-dependent) $B_{\|}$-scale $(\sim 0.5-2$ Tesla) [8]. Such a splitting of ZBA is taken as a distinctive feature of Kondo-type dynamics [1, 2]. The effective $g$-factor $g^{*}$ was found to be both sample- and $n_{\mathrm{s}^{-}}$ dependent $g^{*} / g_{\mathrm{b}} \sim 1-3$, where $\left|g_{\mathrm{b}}\right|=0.44$, consistent with exchange-induced enhancement at low $n_{\mathrm{s}}$,8, 11].

Kondo-type dynamics also lead to a suppression of the ZBA with increasing $T$. Indeed, such a suppression is observed in all our samples as shown in Fig. 2a for nine different $V_{\mathrm{g}}$ 's in T46. In case of single peaks (sets 2,3 , 7 and 8), the suppression is monotonic as $T$ is increased from $\approx 30 \mathrm{mK}$ to $\approx 800 \mathrm{mK}$. For $\Delta \neq 0$, the $T$ dependence of $d I / d V_{\mathrm{SD}}$ is nonmonotonic in $T$ close to $E_{\mathrm{F}}$ (see sets $1,4,5,6$, and weakly in 9 ). Fig. $2 \mathrm{~b}$ shows the $T$ -
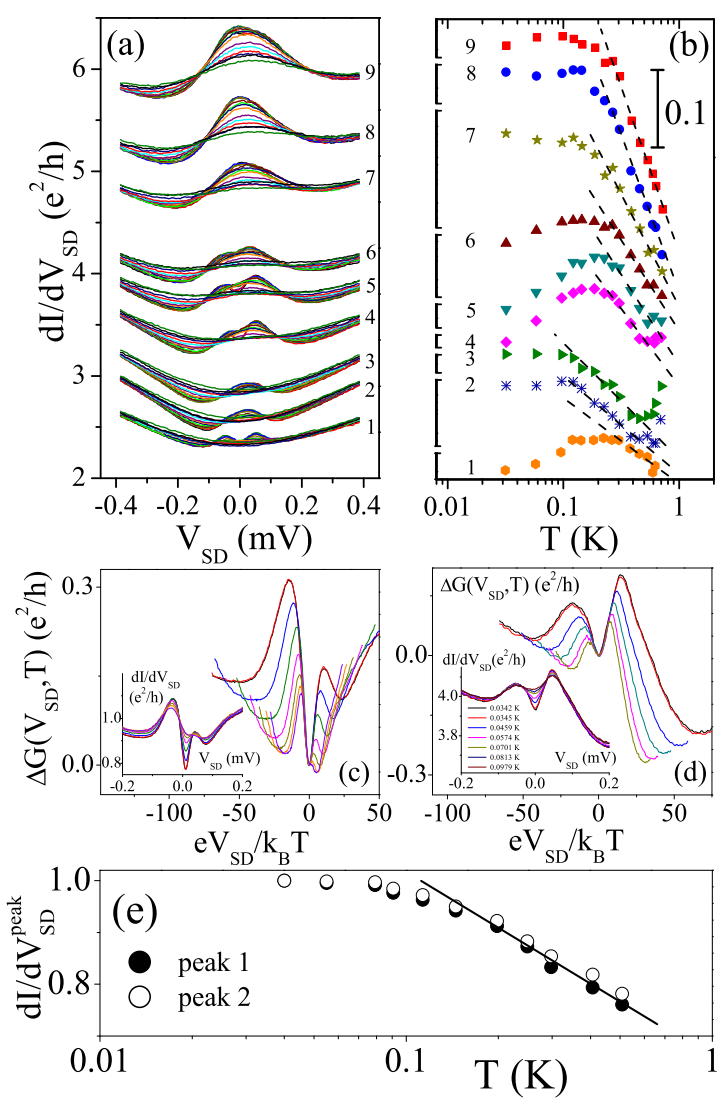

FIG. 2: (Color online) (a) Suppression of the zero-bias anomaly with increasing temperature $(T)$. Each of the nine sets was recorded at a fixed gate voltage $\left(V_{\mathrm{g}}\right)$ and $B_{\|}=0$. (b) Dependence of the linear conductance $G$ on $T$ at different $V_{\mathrm{g}}$. The length of the vertical bar denote $0.1 \times e^{2} / h$. (c),(d) Collapse of $\Delta G$ onto a single trace at low- $V_{\mathrm{SD}}$ as a function of $e V_{\mathrm{SD}} / k_{\mathrm{B}} T$, at two regimes of zero-bias conductance $(G)$. The insets show the unscaled original data. (e) $T$-dependence of individual peaks in a double-peak pattern.

dependence of $G$ from the traces in Fig. 2a. Qualitatively, the high- $T$ regime (typically $T \gtrsim 300 \mathrm{mK}$ ) is similar for all cases, where $G$ shows a "metal"-like decrease with increasing $T$. For $T \lesssim 100-150 \mathrm{mK}$, the increasing behavior of $G$ with $T$ appears intermittently, at values of $V_{\mathrm{g}}$ where $d I / d V_{\mathrm{SD}}$ shows resolvable double-peak structure (Fig. $2 \mathrm{~b}$ and $3 \mathrm{~b}-\mathrm{e}$ ). This reentrant nature is crucial, because even if the high- $T$ suppression of $G$ is attributed to various combinations of phonon contribution, the interaction correction, or the Altshuler-Aronov-type correction from electron scattering by Friedel oscillations [12], the repeated change in the sign of $d G / d T$ is clearly inconsistent with standard weak-localization/interactionbased mechanisms having a single transition from localized to "metallic" state transport. However, in the Zeeman split regime at high $B_{\|}, G$ increases with $T$ at all $V_{\mathrm{g}}$, indicating standard localized state transport.

Most striking aspect of the double-peak structure is the collapse of $\Delta G\left(V_{\mathrm{SD}}, V_{\mathrm{g}}, T\right)=d I / d V_{\mathrm{SD}}\left(V_{\mathrm{SD}}, V_{\mathrm{g}}, T\right)-$ 
$G\left(V_{\mathrm{g}}, T\right)$, onto a single $V_{\mathrm{g}}$-dependent trace when $V_{\mathrm{SD}}$ is scaled by $T$. In Fig. 2(c) and (d) this is illustrated for ZBA's at two different $G$ (and hence $V_{\mathrm{g}}$ ), indicating a common underlying mechanism. The insets contain the actual data prior to substraction of $G\left(V_{\mathrm{g}}, T\right)$, showing the thermal broadening of $d I / d V_{\mathrm{SD}}$, and the suppression of the ZBA splitting with increasing $T$. In Fig. 2e we show the $T$-dependence of individual peaks in $d I / d V_{\mathrm{SD}}$ in the double-peak region at the given $V_{\mathrm{g}}$. For comparison, the individual $T$-dependences are normalized by the $d I / d V_{\mathrm{SD}}$ at lowest $T$. Note the approximately logarithmic $T$-dependence of both peaks at $T \gtrsim 150 \mathrm{mK}$, which is also expected in the Kondo-framework.

The low- $V_{\mathrm{SD}}$ scaling of $d I / d V_{\mathrm{SD}}$ indicates nonequilibrium conductance of the form,

$$
\frac{d I}{d V_{\mathrm{SD}}}\left(V_{\mathrm{SD}}, V_{\mathrm{g}}, T\right)=G\left(V_{\mathrm{g}}, T\right)+A T^{\alpha} \mathcal{F}\left[V_{\mathrm{g}}, \frac{e V_{\mathrm{SD}}}{k_{\mathrm{B}} T}\right]
$$

where $A$ is a phenomenological constant, and $\alpha=0$ gives the best collapse onto the single sample-dependent function $\mathcal{F}\left(V_{\mathrm{g}}, e V_{\mathrm{SD}} / k_{\mathrm{B}} T\right)$. The dependence of $\mathcal{F}$ on $V_{\mathrm{g}}$ will be discussed later. The scaling form of Eq. 1 has been observed in the context of the scattering of electrons from bistable systems [5, 13], and addressed in a twochannel Kondo (2CK) framework 6, 7]. In quasi-ballistic nanostructures, $2 \mathrm{CK}$ dynamics may arise from two possibilities: (1) Electron scattering off systems with quasidegenerate orbital states acting as pseudospins, while the real spins act as the channel index. The nature of this orbital degeneracy can however vary from equivalent lattice defects in metallic nanobridges 13], to singlet-triplet degeneracy in multilevel quantum dots [1, 15]. (2) An underscreened high-spin $(S)$ ground state coupled to $M$ $(<2 S)$ conduction channels [16]. When parametrized in terms of a two-impurity Kondo problem, two spins $S_{1}$ and $S_{2}\left(S=S_{1}+S_{2}\right)$ interact with conduction electrons with antiferromagnetic exchange parameters $J_{1}$ and $J_{2}$ $\left(J_{2} \neq J_{1}\right)$, and a direct exchange $I\left(J_{1}, J_{2} \gg I\right)$ [17]. A two-stage screening process can then decouple the spins from the conduction band by forming $S_{1}-S_{2}$ singlet if $I$ exceeds some critical magnitude. Since both stages can be suppressed by lifting the spin degeneracy with Zeeman energy, a distinctive feature of this mechanism is the nonmonotonicity of $G$ in both $T$ and $B_{\|}$, with a quantitative correspondence between the respective energy scales 16 .

In Fig. 3, we compare the $T$-dependence of $G$ to its $B_{\|\left.\right|^{-}}$ dependence in T45 (on a separate cooldown). Four representative $V_{\mathrm{g}}$, with corresponding nonequilibrium traces at $T \approx 32 \mathrm{mK}$ and $B_{\|}=0 \mathrm{~T}$, are identified as $V_{\mathrm{g} 1}$ to $V_{\mathrm{g} 4}$ in Fig. 3a. Figs. 3b-e show the $\left(B_{\|}=0\right) T$-dependence of $G$ at these $V_{\mathrm{g}} \mathrm{s}$, while Figs. $3 \mathrm{f}$-i show the $B_{\|}$-dependence at the base $T \approx 32 \mathrm{mK}$. We note that apart from the qualitative nonmonotonic behavior of $G$ as a function of both $T$ and $B_{\|}$at $V_{\mathrm{g} 1}$ and $V_{\mathrm{g} 3}$, there is also a quantitative agreement in the energy-scales over which the double-

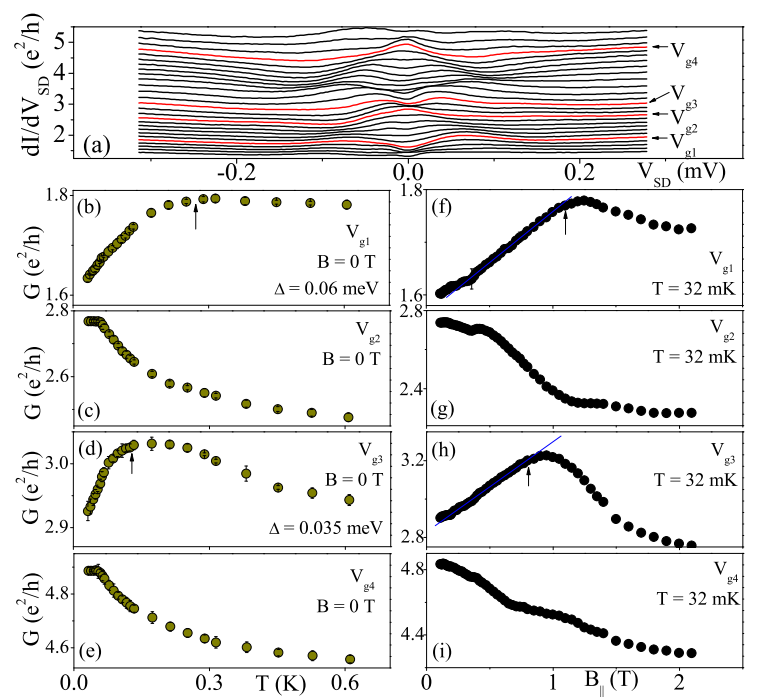

FIG. 3: (Color online) Correspondence between the sourcedrain bias, thermal and Zeeman energy scales: (a) Nonequilibrium traces in T45 identifying two single and two doublepeak regions at gate voltages $V_{\mathrm{g} 1}$ to $V_{\mathrm{g} 4}$. (b)-(e) Temperature $(T)$-dependence of the linear conductance $G$. (f)-(i) In-plane magnetic field $\left(B_{\|}\right)$dependence of $G$ at corresponding $V_{\mathrm{g}} \mathrm{s}$.

peak structures are suppressed. For example, as the half width $\Delta / 2$ reduces from $\approx 0.03 \mathrm{meV}$ at $V_{\mathrm{g} 1}$ to $\approx 0.017$ $\mathrm{mV}$ at $V_{\mathrm{g} 2}$, we find a corresponding decrease in the characteristic thermal and Zeeman energy scales (denoted by the vertical arrows) from $\sim 0.023 \mathrm{meV}$ and $\sim 0.028 \mathrm{meV}$ respectively at $V_{\mathrm{g} 1}$, to $\sim 0.011 \mathrm{meV}$ and $\sim 0.02 \mathrm{meV}$ respectively at $V_{\mathrm{g} 2}$. Since $B_{\|}$is applied in the plane of the 2DES (to an accuracy better than $0.2^{\circ}$ ), conventional weak-localization effects are unlikely to contribute to the observed behavior [19]. Coupling of $B_{||}$to orbital degree of freedom through spin-orbit interaction [20] or finite thickness effect 21] are also excluded since neither antilocalization to weak-localization crossover, nor a stronger suppression of $G$ with increasing $B_{\|}$at lower $n_{\mathrm{s}}$ were observed.

The Kondo-type enhancement in mesoscopic 2D nonlinear transport in the ultra-clean limit indicates an unexpected scattering mechanism of the lead electrons. Moreover, intermittent splitting of the ZBA implies a twostate nature of the scatterer that becomes resolvable only at very low $T(\lesssim 150 \mathrm{mK})$. In view of the quasiballistic nature of transport, the non-equilibrium conductance can represent the tunnelling characteristics between the source and drain across the potential barrier formed by the gate. Presence of localized magnetic states within the barrier region of traditional tunnel junctions has been shown to result in Kondo-type enhancement in the tunnelling conductance 1]. In nanostructures fabricated from MBE-grown high-quality GaAs/AlGaAs heterostructure, localized acceptor sites close to the system can significantly modify the transport through ca- 


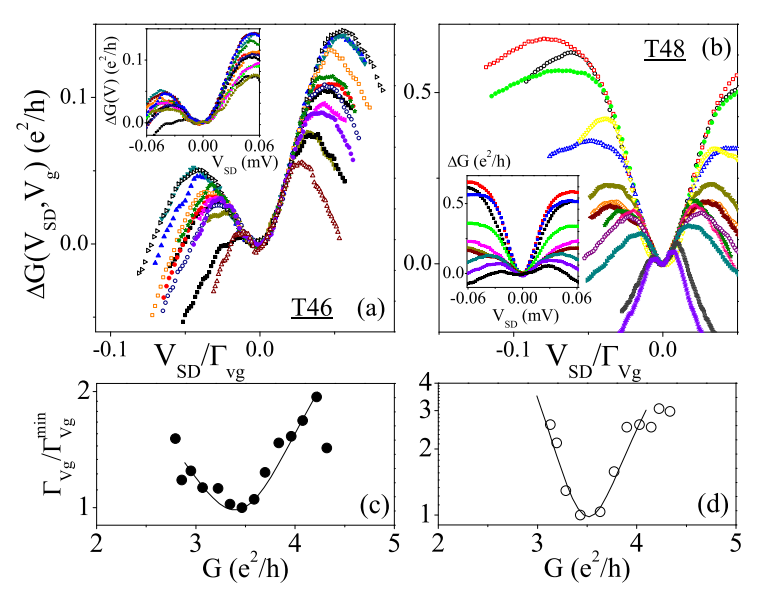

FIG. 4: (Color online) Scaling of the low-energy nonequilibrium characteristics with a gate-voltage $\left(V_{\mathrm{g}}\right)$-dependent energy-scale $\Gamma\left(V_{\mathrm{g}}\right)$ at $T \approx 32 \mathrm{mK}$ for (a) T46 and (b) T48. Insets show the unscaled, but zero-shifted, traces. Both sets were obtained for $G$ around $4 e^{2} / h$ in Fig. 1. (c),(d) Variation of $\Gamma\left(V_{\mathrm{g}}\right)$ as a function linear conductance $G$ in the T46 and T48 respectively. Solid lines are guide to the eye.

pacitive coupling 22. This could lead to a Kondoassisted tunnelling, similar to that observed in metallic nanobridges [23], and also in semiconductor-metal junctions 24]. Arguments against such a scenario are, (1) reproducibility, and insensitivity of the relevant energy scales to sample-specific details, and (2) enhancement in the amplitude of ZBA at lower disorder.

Alternatively, dependence of the effect on $n_{\mathrm{s}}$, i.e. relatively strong amplitude of ZBA in samples with low- $n_{\mathrm{s}}$, and also weakening of the effect with increasing $n_{\mathrm{s}}$ within a given sample, indicates a possible role of Coulomb interaction. Typically in our systems, the low magnitude of $n_{\mathrm{s}}\left(\sim 0.8-3 \times 10^{10} \mathrm{~cm}^{-2}\right)$ results in a large interaction parameter, $r_{\mathrm{s}}=1 / a_{\mathrm{B}} \sqrt{\pi n_{\mathrm{s}}} \sim 3.5-6$, where $a_{\mathrm{B}}$ is the effective Bohr radius. This corresponds to an exchange energy that can lead to strong many-body spin fluctuations with magnetic moment $\gg 1 / 2$ 25]. Underscreening of such spin fluctuations when Kondo-coupled to lead electrons can result in split Kondo resonance, which would be strongly $V_{\mathrm{g}}$-dependent. Exchange splitting of Kondo-resonance in presence of itinerant electron ferromagnetism has been observed experimentally [26]. Here, the suppression of the double peak pattern in $B_{\|}$ provides further evidence of the dynamics to be related to real spin, rather than pseudospin resulting from quasidegenerate orbital states. Indeed, similar ZBA observed in quasi-1D quantum point contacts have been interpreted in terms of a dynamic spin-polarization of the electrons within the $1 \mathrm{D}$ channel [4].

Finally, we discuss the intermittent collapse of $\Delta$ observed in Fig. 1. If the scaling relation of Eq. 10 1 originates from proximity of a $2 \mathrm{CK}$ fixed point [5, 13], the low- $T$ $V_{\mathrm{g}}$-dependence of $\Delta$ indicates that such scaling should also be possible in terms of a $V_{\mathrm{g}}$-dependent energy-scale $\Gamma\left(V_{\mathrm{g}}\right)$. This implies an asymptotic form of the scaling function $\mathcal{F} \rightarrow \mathcal{F}\left[V_{\mathrm{SD}} / \Gamma\left(V_{\mathrm{g}}\right)\right]$ as $T \rightarrow 0$. Theoretically, $\Gamma$ is analogous to the energy asymmetry in case of orbital degeneracy, or the direct exchange parameter $I$ in the two-impurity Kondo parametrization 15, 16, 17, 18], which depends implicitly on $V_{\mathrm{g}}$ through the exchange interaction magnitude $J$ 27]. Indeed, in Fig. 4a and 4b we have shown the low-energy scaling of $\Delta G\left(V_{\mathrm{SD}}, V_{\mathrm{g}}\right)=$ $d I / d V_{\mathrm{SD}}\left(V_{\mathrm{SD}}, V_{\mathrm{g}}\right)-G\left(V_{\mathrm{g}}\right)$ in $\mathrm{T} 46$ and $\mathrm{T} 48$ at the base $T \approx 32 \mathrm{mK}$. In the absence of an absolute scale, we have expressed $\Gamma\left(V_{\mathrm{g}}\right)$ with respect to its minimum value $\left(\Gamma\left(V_{\mathrm{g}}\right)^{\mathrm{min}}\right)$ observed around $G \sim 3.5 \times e^{2} / h$ in both samples (Fig. $4 \mathrm{c}$ and $4 \mathrm{~d}$ ). The scalability of $\Delta G$ close to the collapse of $\Delta$ strongly indicates the possibility of a quantum critical dynamics. However, a satisfactory explanation of its reentrant nature and the fundamental mechanism of the 2CK-type scattering forms the basis of ongoing investigations.

A.G. acknowledges fruitful discussions with G. Gumbs and V. I. Fal'ko. This work was supported by an EPSRC funded project. C.S. acknowledges financial support from Gottlieb Daimler- and Karl Benz-Foundation.

[1] L. Y. L. Shen and J. M. Rowell, Phys. Rev. 165, 566 (1968); P. Nielsen, Phys. Rev B 2, 3819 (1970); S. Bermon, D. E. Paraskevopoulos and P. M. Tedrow, ibid. 17, 2110 (1978).

[2] D. Goldhaber-Gordon et al., Nature 391, 156 (1998); S. M. Cronenwett, T. H. Oosterkamp and L. P. Kouwenhoven, Science 281, 540 (1998).

[3] N. S. Wingreen and Y. Meir, Phys. Rev. B 49, 11040 (1994).

[4] S. M. Cronenwett et al., Phys. Rev. Lett. 88, 226805 (2002).

[5] D. C. Ralph and R. A. Buhrman, Phys. Rev. B 51, 3554 (1995).

[6] A. W. W. Ludwig and I. Affleck, Phys. Rev. Lett. 67, 3160 (1991); I. Affleck and A. W. W. Ludwig, Phys. Rev. B 48, 7297 (1993).

[7] K. A. Matveev, Phys. Rev. B 51, 1743 (1995).

[8] A. Ghosh, C. J. B. Ford, M. Pepper, H. E. Beere, and D. A. Ritchie, Phys. Rev. Lett. 92, 116601 (2004).

[9] B. L. Altshuler and A. G. Aronov, in Electron-Electron Interaction in Disordered Systems, edited by A. L. Efros and M. Pollak (North-Holland, Amsterdam, 1985); A. M. Finkel'stein, Sov. Phys. JETP 57, 97 (1983).

[10] The ZBA becomes suppressed in a sample-dependent manner at stronger background disorder in systems with thinner spacers $(\lesssim 40-60 \mathrm{~nm})$, or larger sample dimensions $(L \gtrsim 10 \mu \mathrm{m})$, or at low- $n_{\mathrm{s}}$ localized regime where $G \ll 2 e^{2} / h$ with weak disorder screening.

[11] J. Zhu et al., Phys. Rev. Lett. 90, 056805 (2003); E. Tutuc, S. Melinte and M. Shayegan, ibid. 88, 036805 (2002).

[12] Y. Y. Proskuryakov et al., Phys. Rev. Lett. 89, 076406 (2002); G. Zala, B. N. Narozhny and I. L. Aleiner, Phys. Rev. B 64, 214204 (2001). 
[13] D. C. Ralph, A. W. W. Ludwig, J. von Delft and R. A. Buhrman, Phys. Rev. Lett. 72, 1064 (1994).

[14] W. G. van der Wiel et al., Phys. Rev. Lett. 88, 126803 (2002).

[15] W. Hofstetter and H. Schoeller, Phys. Rev. Lett. 88, 016803 (2002).

[16] M. Pustilnik and L. I. Glazman, Phys. Rev. Lett. 87, 216601 (2001).

[17] M. Vojta, R. Bulla, and W. Hofstetter, Phys. Rev. B 65, 140405 (2002); I. Affleck, A. W. W. Ludwig, and B. A. Jones, ibid. 52, 9528 (1995); K. Kikoin and Y. Avishai, Phys. Rev. Lett. 86, 2090 (2001); P. Nozières and A. Blandin, J. Phys. (Paris) 41, 193 (1980).

[18] M. Pustilnik, L. Borda, L. I. Glazman, and J. von Delft, Phys. Rev. B 69, 115316 (2004).

[19] P. A. Lee and T. V. Ramakrishnan, Rev. Mod. Phys
57, 287 (1985); G. Bergmann, Phys. Rev. Lett. 48, 1046 (1982).

[20] J. A. Folk et al., Phys. Rev. Lett. 86, 2102 (2001); I. L. Aleiner and V. I. Fal'ko, ibid. 87, 256801 (2001).

[21] S. Das Sarma and E. H. Hwang, Phys. Rev. Lett. 84, 5596 (2000).

[22] D. H. Cobden et al., Phys. Rev. B 44, R1938 (1991).

[23] D. C. Ralph and R. A. Buhrman, Phys. Rev. Lett. 72, 3401 (1994).

[24] E. L. Wolf and D. L. Losee, Phys. Rev. B 2, 3660 (1970).

[25] D. Varsano, S. Moroni, and G. Senatore, Europhys. Lett. 53, 348 (2001); M. W. C. Dharma-wardana and F. Perrot, Phys. Rev. Lett. 90, 136601 (2003).

[26] A. N. Pasupathy et al., Science 306, 86 (2004).

[27] S. Tarucha et al., Phys. Rev. Lett. 84, 2485 (2000). 